\title{
From the Editors' Desk: Physician Heal Thyself
}

\author{
Mitchell D. Feldman, MD, MPhil \\ Division of General Internal Medicine, Department of Medicine, University of California, San Francisco, San Francisco, CA, USA.
}

$\mathrm{J}$ Gen Intern Med 26(8):823

DOI: $10.1007 / \mathrm{s} 11606-011-1755-0$

(c) Society of General Internal Medicine 2011

$\mathrm{N}$ umerous studies have documented high rates of job dissatisfaction and burnout among physicians. Many factors have contributed to this trend including the rise of managed care and the concomitant increased paperwork and other administrative demands that sap time that could be spent with patients and leave physicians with a diminished sense of autonomy and control over their work. Physicians who work at academic medical centers face the additional challenge of a shrinking federal budget for research and increasing demands on their time for "unfunded mandates" such as teaching and mentoring. The end result has been unacceptably high rates of burnout, defined as a sense of emotional exhaustion, cynicism and a reduced sense of effectiveness, or as the pioneering researchers on burnout Maslach and Leiter pointedly write in their book The Truth About Burnout: "(Burnout) represents an erosion in values, dignity, spirit, and will-an erosion of the human soul."

The impact of physician burnout can be significant both for the individual and for the organization in which they work. Decreased productivity, poor retention, medical errors and negative patient interactions are just some of the documented potential consequences of burnout among physicians both for the individuals and for the organization. For the physicians themselves, depression, substance abuse and impaired relationships with family and friends may be the result of untreated stress and eventual burnout. But with these challenges come opportunities for physicians to enhance their well-being and avoid burnout. Well-being arises, in part, when we take the time to clarify our personal values and make career and personal choices that honor those values.

Well-being is not just a personal endeavor, however; physician well-being is also an organizational and political

Published online June 7, 2011 issue. The personal commitment of physicians to healthy values and behaviors may be eroded in organizations that reward overwork and dissociation from human feelings and meaning at work. In this issue of JGIM, two articles address the issue of burnout and well-being among physicians and trainees from an organizational perspective. Sood et al. report on a small, randomized trial of a stress management and resiliency-training program (SMART) for department of medicine physicians. They found that compared with a wait-list control arm, physicians randomized to a single 90-minute oneon-one session focusing on meditation, relaxation and "attention and interpretation" had significantly decreased perceived stress, anxiety and improved overall quality of life at 8 weeks. While the data is limited, this pilot study suggests that introducing more stress management training programs into academic health centers could enhance resiliency and decrease burnout amongst academic physicians.

One well-known contributor to physician stress and burnout is overwork. In this issue, a systematic review by Fletcher et al. examines the impact of the 2003 duty hour reform on resident burn-out and well-being (among other resident and patient centered outcomes). Overall, they found an improvement in resident well-being following the mandated decrease in work hours after the 2003 duty hour reform (but perhaps surprisingly, no impact on rates of depression). While duty hour reform has certainly helped to promote better self-care among residents, other studies have found that trainees are still susceptible to unacceptably high rates of burnout. Reducing work hours is a necessary first step to combat burnout among our trainees, but clearly it is not enough. We must teach the next generation of physicians to focus more on self-care including their physical, mental and emotional well-being. This is essential for the individual physicians and for the patients they serve.

Corresponding Author: Mitchell D. Feldman, MD, MPhil; Division of General Internal Medicine, Department of Medicine, University of California, San Francisco, 1545 Divisadero St., San Francisco, CA 94143-0320, USA (e-mail: mfeldman@medicine.ucsf.edu). 